J. PHYS. C (PROC. PHYS. SOC.), 1968, SER. 2, VOL. 1. PRINTED IN GREAT BRITAIN

\title{
The temperature dependence of the $F$ band in magnesium oxide
}

\author{
B. HENDERSON, $\uparrow$ R. D. KING + and A. M. STONEHAM $\S$ \\ $\dagger$ Physics Department, Lniversity of Keele, Keele, Staffs. \\ $\doteqdot$ Ceramics Division, Atomic Energy Research Establishment, Harwell, Didcot, \\ Berks. \\ $\S$ Theoretical Physics Division, Atomic Energy Research Establishment, Harwell, \\ Didcot, Berks.
}

MS. received 9th January 1968, in revised form 20 th February 1968

\begin{abstract}
The position and width of the $F$ band in magnesium oxide have been measured in the temperature range $4-400^{\circ} \mathrm{K}$. The data have been analysed in terms of the simplest adequate 'configuration coordinate' model. The width results give an effective frequency of $7.8 \times 10^{12} \mathrm{~s}^{-1}$, which is close to a peak in the phonon density of states and to the value extrapolated from data for the alkali halides. There is evidence that the effective frequency is reduced by about $5 \%$ in the excited state of the F centre. The Huang-Rhys factor is about 39 , and luminescence is predicted at about $2.4 \mathrm{ev}$. The band shape indicates the existence of three small absorption bands on the high-energy side of the main $F$ absorption band. These appear to be associated with the $F$ centre, but their nature is not clear.
\end{abstract}

\section{Introduction}

Wertz et al. (1957) first demonstrated that $F$ centres are present in neutron-irradiated magnesium oxide, and the ground-state magnetic properties of this defect are now well understood, as a result of subsequent work (Wertz et al. 1961, Unruh and Culvahouse 1967). By contrast, less is known of the optical properties of the centre although it is now established that the F band occurs at $4.9 \mathrm{ev}$ (Henderson and King 1966, Wertz et al. 1964, Kemp et al. 1966). Details of its structures are, however, hard to obtain largely as a result of the transition metal impurities in commercially available single crystals. These impurities have absorption bands in the ultra-violet and visible regions of the spectrum and obscure the absorption by intrinsic lattice defects.

We report the temperature dependence of the width and peak position of the $\mathrm{F}$ band in the temperature range $4-400^{\circ} \mathrm{K}$. The neutron irradiation conditions and dose were chosen so that the F-centre absorption dominated in the ultra-violet region of the spectrum. Several impurities, notably $\mathrm{Fe}^{3+}$, also gave a significant optical absorption, so the various overlapping bands were resolved by a computer technique. New weak bands appear to be present, and their origin is also discussed. The width and shift of the main $(4.9 \mathrm{ev})$ band are then analysed in terms of the simplest models which give behaviour similar to that observed. Using these models, we estimate the Huang-Rhys factor (the mean number of phonons emitted in absorption) and the expected position of the F-centre luminescence.

\section{Experimental details}

The single crystals used were purchased from the Norton Co., Worcester, Mass., U.S.A. A typical analysis, reported previously (Henderson and King 1966), showed iron to be the most important impurity for the present purposes. Iron existing as $\mathrm{Fe}^{3+}$ in magnesium oxide gives two bands at $4 \cdot 3 \mathrm{ev}$ and $5 \cdot 7 \mathrm{ev}$ (Soshea et al. 1958).

The single crystals were cleaved to appropriate dimensions, then irradiated in the Harwell PLUTO reactor at $45^{\circ} \mathrm{C}$. The work reported here was confined to a sample irradiated to a dose of $3.22 \times 10^{17}$ neutrons $\mathrm{cm}^{-2} \uparrow$ Neutron irradiation produces relatively large concentrations of $F$ centres: the present sample had a concentration of the order of $10^{18} \mathrm{~cm}^{-3}$. In the range below $1 \times 10^{18}$ neutrons $\mathrm{cm}^{-2}$, the shape and width of the $\mathrm{F}$ band

$\dagger$ All doses are quoted for neutrons with energy above $1 \mathrm{mev}$. 
are essentially independent of the fast-neutron dose. At higher doses other bands are observed overlapping the F band (Henderson and King 1966). These bands may be related to the defect clusters detected earlier using paramagnetic resonance (Henderson 1966, Wertz et al. 1961). The F-centre concentration was, however, sufficiently high for the $\mathrm{F}$ band to dominate in the ultra-violet region of the spectrum.

After irradiation the sample was polished in hot $\left(130^{\circ} \mathrm{C}\right)$ orthophosphoric acid, diluted in the ratio $1: 5$, to remove undesirable surface features and to obtain a suitable thickness for optical absorption measurements. These measurements were made with a Cary 14 spectrophotometer operating in the range 1860-25000 $\AA$ and recording absorbances up to 5 by using neutral density filters. No automatic digital output was available, so absorbance values were read at $10 \AA$ intervals directly from the chart recording during a very slow scan. The measurements were made at thirteen temperatures in the range $4-400^{\circ} \mathrm{K}$ with a metal cryostat having quartz windows.

\section{Analysis of the absorption spectrum}

The observed absorption spectrum consisted of several overlapping bands. The data analysis was made in two stages: an approximate resolution using a curve resolver, and an accurate resolution of the component bands by computer methods.

In the approximate resolution the known spectral characteristics of impurity bands were used, assuming that they all had a Gaussian shape. These bands were the $4 \cdot 3 \mathrm{ev}$ and $5.7 \mathrm{ev}$ bands due to $\mathrm{Fe}^{3+}$ (Soshea et al. 1958) and the $6.8 \mathrm{ev}$ band (Johnson 1954). It proved necessary to include three additional weak bands in the range 5.3-5.9 ev. Two of these bands show up as slight inflections on the side of the $\mathrm{F}$ band; all three are needed to account for the rise in absorption on the high-energy side of the $\mathrm{F}$ band. We shall discuss the evidence for the existence of these bands and their possible origin later.

The approximate resolution gave estimates of the position, intensity and half-width of the component bands. These data were used as the computer input for accurate resolution of the spectra. The programme used was $(\mathrm{M} \phi \mathrm{RC} \phi \mathrm{S})$, developed and tested by Biggers et al. (1966). It uses an iterative non-linear procedure to minimize the sums of the squares of the differences between the experimental data and the data calculated from a trial resolution of the spectrum.

The resolved spectrum at $4^{\circ} \mathrm{K}$ is shown in figure 1 , and the band parameters from the computer analysis are given in table 1 . The error analysis shows that the errors are greatest

\section{Table 1. Band parameters from the computer resolution of the absorption spectrum at $4{ }^{\circ} \mathrm{K}$}

$\begin{array}{lccc}\text { Band } & \begin{array}{c}\text { Peak } \\ \text { position (ev) }\end{array} & \begin{array}{c}\text { Half- } \\ \text { width (ev) }\end{array} & \begin{array}{c}\text { Oscillator } \\ \text { strength }\end{array} \\ 1\left(\mathrm{Fe}^{3+}\right) & 4.30 & 0.730 & \\ 2 \mathrm{~F} \mathrm{band} & 4.95 & 0.477 & 0.80 \dagger \\ 3 & 5.31 & 0.23 & 0.15 \dagger \\ 4 & 5.58 & 0.305 & 0.08 \dagger \\ 5\left(\mathrm{Fe}^{3+}\right) & 5.62 & 0.960 & \\ 6 & 5.94 & 0.73 & 0.24 \dagger\end{array}$

† Calculated relative to the F-centre concentration, which is assumed equal to the concentration of defects responsible for the bands.

See Henderson and King (1966).

on the high-energy side of the $\mathrm{F}$ band as expected, and the spectral analysis to be a very good fit to the experimental data. No change in this fit is observed if the F-band peak is allowed to shift by $\pm 0.005 \mathrm{ev}$; for the weaker bands at higher energies a shift of less than $\pm 0.012 \mathrm{ev}$ is allowed. 
The bands at $\mathbf{5} \cdot 30 \mathrm{ev}, \mathbf{5} \cdot \mathbf{5 8} \mathrm{ev}$ and $\mathbf{5} \cdot 95 \mathrm{ev}$ are weak and have not been reported before. They do not appear to be a result of the use of Gaussian line shapes for the bands; it is clear that no simple smooth shape would give the inflections in the absorption due to the bands at 5.30 and $5.58 \mathrm{ev}$. In addition, the analysis seems to require these bands up to the

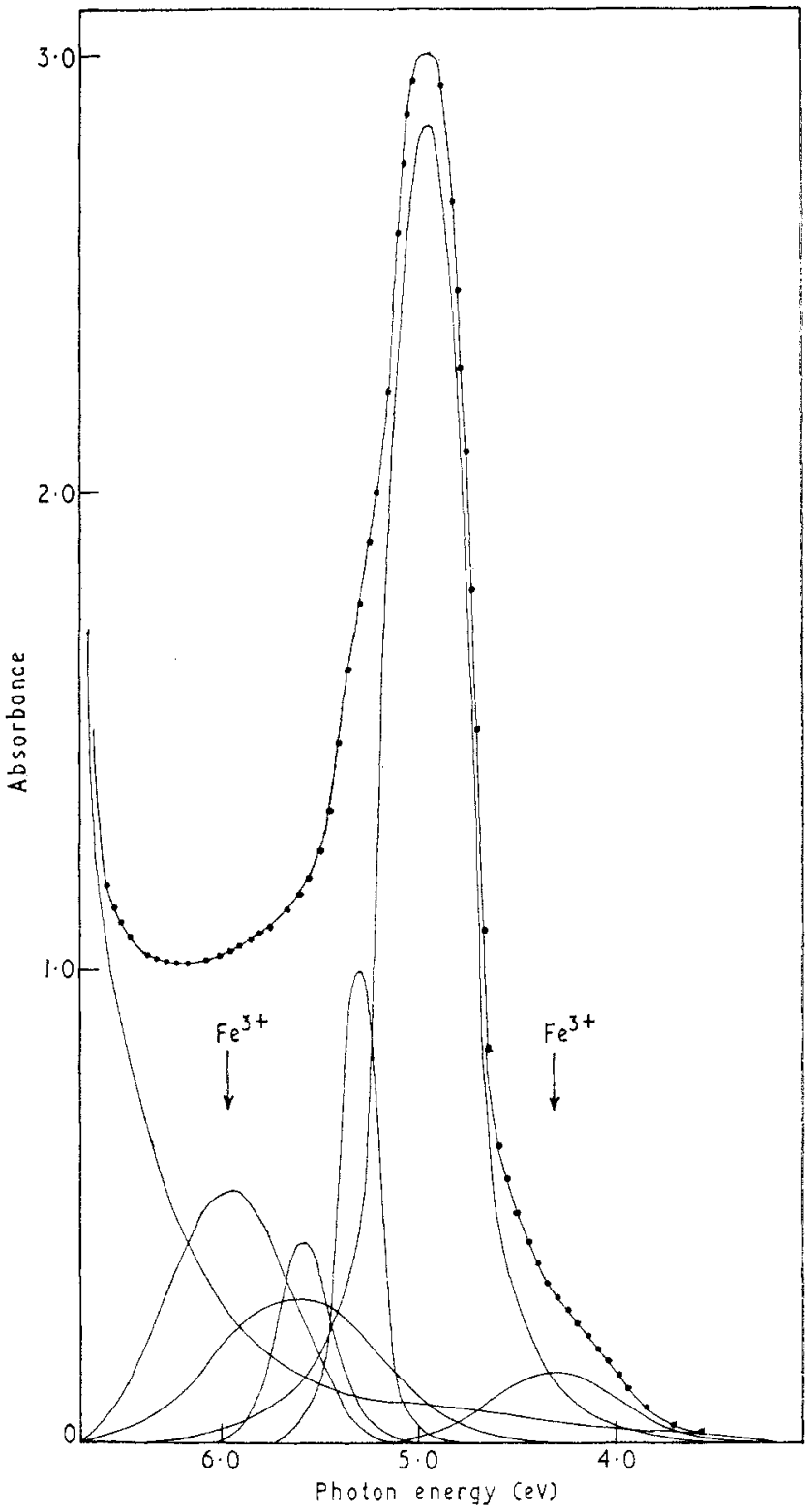

Figure 1. Showing the ultra-violet absorption at $4{ }^{\circ} \mathrm{K}$ of magnesium oxide single crystals irradiated with fast neutrons to a dose of $3.22 \times 10^{17}$ neutrons $\mathrm{cm}^{-2}$. The band at $4.9 \mathrm{ev}$ is the $\mathrm{F}$ band; the weaker bands are obtained from a computer analysis of the absorption shape, after subtracting the absorption due to bands at $6 \cdot 8 \mathrm{ev}, 5.7 \mathrm{ev}\left(\mathrm{Fe}^{3+}\right)$ and $4.3 \mathrm{ev}\left(\mathrm{Fe}^{3+}\right)$ as discussed in the text.

highest temperatures, when Gaussians should be good approximations. It seems probable that irradiation bands may be present in the crystals in the range $6.0-6.8 \mathrm{ev}$; these possibilities have not been investigated although it is realized that the presence of such bands will have important consequences upon the analysis above $5.5 \mathrm{ev}$. Resolution of the $\mathrm{F}$ band 
should not be materially affected by such bands. The weak bands are absent in unirradiated crystals or in electron- or $\gamma$-irradiated crystals, so it seems unlikely that they come from impurities. It seems more plausible that they are associated with the $F$ centre, possibly transitions of the F-centre electron to higher excited states. Such transitions are quite well understood in the alkali halides (Lüty 1960, Spinolo and Smith 1965). Another possibility is that the bands are due to $F$-centre aggregates, or to $F$ centres perturbed by the presence of near-neighbour defects. This would be consistent with the earlier observation of bands at these positions in samples irradiated to high dose (Henderson and King 1966).

\section{Temperature dependence of the $F$ band}

In this section we describe the temperature dependence of the half-width of the $F$ band, $H(T)$, and the position of its peak, $E(T)$. The measured values of $H(T)$ and $E(T)$ are shown in figure 2. We then interpret these quantities in terms of the simplest model capable

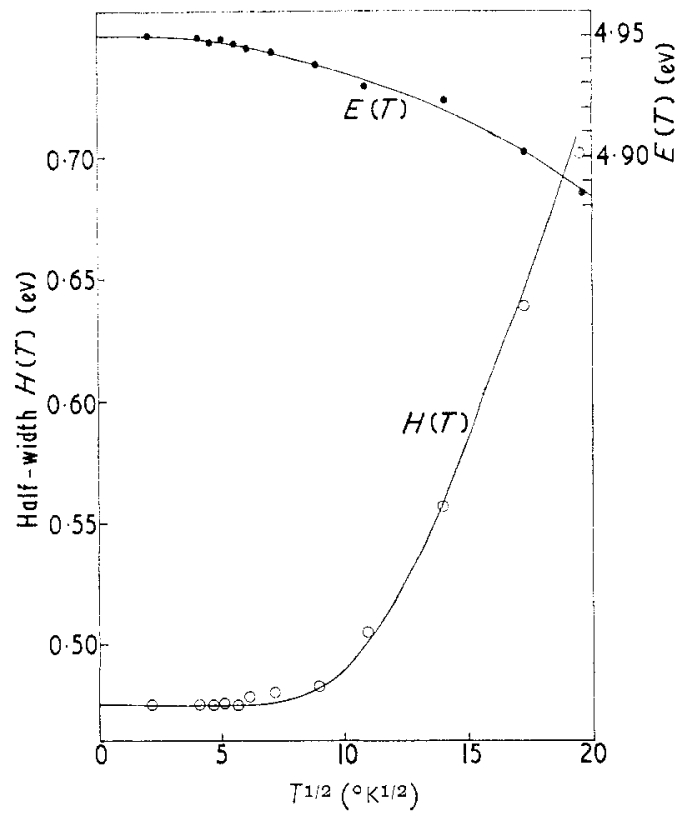

Figure 2. The half-width of the $\mathrm{F}$ band $H(T)$ and the position of its peak $E(T)$ plotted as a function of the square root of abolute temperature for single crystals of Norton magnesium oxide.

of fitting the data, the so-called configuration coordinate model (for references see Markham 1966). The assumptions are these. First the interaction of the F centre with the lattice can be approximated by the interaction of the $F$ centre by one effective vibrational mode. This does not imply that the mode is a local mode or a resonant mode, nor is it meant to suggest that only a small fraction of the phonon modes in the crystal interact with the colour centre. It does imply that the effects of all modes are qualitatively well represented by a single mode. In special cases, this approximation is particularly plausible, as in the model of Huang and Rhys (1950) where only longitudinal optic modes interact with the $F$ centre. Secondly, when predicting emission from the $F$ centre we assume that the same mode is still the only one of importance. For the $F$ centre the lattice modes of importance are considered to be those completely symmetric $(A)$ at the $F$ centre. In the excited state, modes of lower symmetry $\left(T_{1}, T_{2}\right.$ or $\left.E\right)$ may become more important because of the Jahn-Teller effect; these we neglect, although we allow the ground- and excited-state frequencies to differ. These low-symmetry modes probably affect the intensity of the emission band more than its shape. Thirdly, a simple model, like a configuration coordinate model, presumes a simple relation between the moments of an absorption 
line and the features usually measured (area, width, peak position, etc.); thus the theoretical predictions are usually based on the moments. However, the present experiments have too high a background for moments to be measured with accuracy, and the width and peak position are obtained instead. 'Thus the present experiments give no information which requires knowledge of the detailed structures of the $F$ band.

Our results give information on the strength of the interaction of the F centre with the 'effective' mode (measured by the Huang-Rhys factor $S$ ) and the frequency of this mode which may be compared with the phonon density-of-states curves for the perfect magnesium oxide lattice, and with the results of similar work on alkali halides. 'These results imply which class of phonon modes is most important, and allow comparison of the electronphonon interaction in oxides with those in alkali halides. The data are also used to predict the luminescence of the $\mathrm{F}$ centre in magnesium oxide.

\subsection{The width of the $F$ band in magnesium oxide}

Interactions of the centre with the lattice modes broaden the absorption band. The temperature dependence of the half-width $H(T)$ is given by

$$
H(T)^{2}=H(0)^{2} \operatorname{coth} \frac{h \bar{\nu}}{2 k T}
$$

where $\bar{v}$ is the effective frequency. The parameters are deduced from a least-squares fit of the data, giving

and

$$
\begin{aligned}
H(0) & =(0.477 \pm 0.002) \mathrm{ev} \\
\bar{\nu} & =(7 \cdot 8 \pm 0 \cdot 1) \times 10^{12} .
\end{aligned}
$$

The deviations from (1) are all less than the estimated experimental error of $\pm 0.005 \mathrm{ev \text {; }}$ although a slight improvement is achieved by introducing a second effective frequency (two-mode model) the change is not significant. The error quoted for $\bar{\nu}$ indicates the changes which can be made in $\bar{v}$ whilst still giving an acceptable fit. It is perhaps worth remarking that the effective frequency is not sensitive to the analysis described in $\S 3$. If the ultra-violet spectrum was treated as one single band plus background (instead of four bands plus impurity bands plus background) the effective frequency is only slightly larger, of order $10 \times 10^{12} \mathrm{~s}^{-1}$. A very simple comparison of magnesium oxide with the alkali halides can be made from the empirical rule of Podini and Spinolo (1966)

$$
2 \pi \bar{\nu}\left(10^{13} \mathrm{~s}^{-1}\right) \beta\left(10^{12} \text { barye }^{-1}\right) \simeq \text { constant }
$$

in which $\beta$ is the compressibility. The constant is 10 for the alkali halides; for magnesium oxide it is $10 \cdot 8$.

$\bar{\nu}$ lies in the acoustic branches of the experimental phonon density of states just below a peak (Peckham 1967). A very similar behaviour is found in a number of alkali halides (Hughes et al. 1967), notably lithium fluoride, sodium fluoride, sodium chloride and potassium chloride. The resemblance of lithium fluoride and magnesium oxide is particularly striking (see table 2) and reflects their similar lattice dynamics (Barron et al. 1959). Other alkali halides with larger atomic masses (e.g. KBr, KI) tend to have effective frequencies lying within the optic phonon branch.

Table 2. Comparison of the optical properties of the MgO and LiF F centres

Effective frequency ground state

Effective frequency excited state

Half-width (ev at $\left.0{ }^{\circ} \mathrm{K}\right)$

Peak position (ev at $0^{\circ} \mathrm{K}$ )

Huang-Rhys factor $S$

Zero-phonon transition energy (ev)

Estimated emission energy (ev)

\begin{tabular}{ll}
$\quad \mathrm{MgO}$ & \multicolumn{1}{c}{$\mathrm{LiF}$} \\
$7.8 \times 10^{12}$ & $8.65 \times 10^{12}$ \\
$7.4 \times 10^{12}$ & \\
0.477 & 0.579 \\
4.95 & 5.10 \\
39 & 41 \\
3.65 & \\
2.4 &
\end{tabular}

The LiF data are from Rabin and Reich (1964). 
There is no evidence that broadening mechanisms other than the electron-phonon are important. We estimate the contributions to $H(0)$ of strain broadening (Stoneham 1966) and isotope effects (Rabin and Reich 1964) to be of the order of $1 \times 10^{-3} \mathrm{ev}$ and $0.5 \times 10^{-3} \mathrm{ev}$, respectively. Both are negligible, as the experimental errors are quite typically $5 \times 10^{-3} \mathrm{ev}$.

We can also calculate the Huang-Rhys factor $S$. This is the mean number of phonons emitted in absorption, and it gives a measure of the interaction of the $F$ centre with the lattice. When only one mode is important, $S$ can be found from Markham's (1959) equation:

$$
S=\left\{\frac{H(0)}{2 \cdot 36 h \bar{\nu}}\right\}^{2} .
$$

In using this equation, we have ignored differences in effective frequency in the ground and excited states $(\$ 4.2)$ and the possibility of two effective frequencies mentioned above. The value of $S$ is not very sensitive to these sophistications, and, in any case, the accuracy of corrections is low. We find

$$
S \simeq 39 \text {. }
$$

This value falls within the range of those found for the alkali halides (Hughes et al. 1967). As the fractional intensity in the zero-phonon transition at $0^{\circ} \mathrm{K}$ is of order $\exp (-S)$, it is clear that this line will not be seen in magnesium oxide.

\subsection{Peak position of the $F$ band}

As the temperature increases the $\mathrm{F}$ band shifts to lower energies. Two effects contribute to this: thermal expansion of the lattice and the differences in effective frequency between the ground and excited states of the $F$ centre. The experiments were made at constant pressure, so we write

$$
\left.\frac{\partial E}{\partial T}\right|_{P}=\left.\left.\frac{\partial E(a, T)}{\partial a}\right|_{T} \frac{\partial a}{\partial T}\right|_{P}+\left.\frac{\partial E(a, T)}{\partial T}\right|_{P} .
$$

$a$ is taken to be the lattice parameter, although strictly it should be a length parameter involving such factors as lattice relaxation around the defect.

The first term in (2) describes the effect of thermal expansion of the lattice, $(\partial a / \partial T)_{P}$. In magnesium oxide the expansion has been measured by Durand (1936). The other factor $(\partial E / \partial a)_{T}$ should be found from the shift of the $F$ band under hydrostatic pressure (cf. Jacobs 1954). No such measurements are available. For a rough estimate we use an IveyMollwo relation for the $\mathrm{F}$ band in magnesium oxide, calcium oxide and strontium oxide:

$$
E(a, T) a^{n}=\text { constant }
$$

from which we obtain the estimate

$$
\left.\frac{\partial E(a, T)}{\partial a}\right|_{T}=-n \frac{E(a, T)}{a(T)} .
$$

The second term in (2) comes from the change in effective frequency in the excited state. At high temperatures, for example (Pryce 1966),

$$
\left.\lim _{T \rightarrow \infty} \frac{\partial E(a, T)}{\partial T}\right|_{P}=f k
$$

where $k$ is Boltzmann's constant and $f$ the fractional change in force constant, or (assuming no change in effective mass)

$$
f=\left(\frac{\bar{\nu}_{\mathrm{exc}}}{\bar{\nu}}\right)^{2}-1
$$

At lower temperatures $(\partial E(a, T) / \partial T)_{P}$ has a $\operatorname{coth}(h \bar{\nu} / 2 k T)$ temperature dependence. 
As the approximations are somewhat crude the results are mainly of qualitative interest. We find from a least-squares fit to the shift, after subtracting the effects of lattice expansion,

$$
\begin{aligned}
\bar{\nu}_{\mathrm{exc}} & \sim 7.4 \times 10^{12} \mathrm{~s}^{-1} \\
\bar{\nu} & \sim 7.8 \times 10^{12} \mathrm{~s}^{-1} .
\end{aligned}
$$

The effective frequency is lower in the excited state than in the ground state. The effects of thermal expansion and the change of force constant shift the $\mathrm{F}$ band in the same direction. In view of some of the approximations we have made, it is not clear how accurate $\bar{\nu}_{\text {exc }}$ is. The residuals of the least-squares fit suggest $\pm 0.2 \times 10^{12} \mathrm{~s}^{-1}$, i.e. the change is significant.

\subsection{Luminescence from the $F$ centre in $M g O$}

We may use our results from the absorption spectrum to predict whether strong luminescence should be observed, and the energy at which it should be most intense.

A criterion for strong luminescence has been proposed by Dexter et al. (1955). The ground-state and excited-state energies, as functions of the configuration coordinate, may intersect at an energy $E_{\mathrm{C}}$ above the minimum in the ground-state energy. In absorption, the transitions in which the configuration coordinate is fixed at the ground-state minimum have an energy $E_{\mathrm{B}}$. Luminescence is expected if $E_{\mathrm{C}}>E_{\mathrm{B}}$. We find this inequality to hold, so strong luminescence is expected, at least in pure crystals.

The energy of the emission can also be estimated from our data. If we ignore the difference in the ground- and excited-state frequencies,

$$
\begin{aligned}
E_{\mathrm{em}} & =E_{\mathrm{abs}}-2 S h \bar{\nu} \\
& =2 \cdot 4 \mathrm{ev} .
\end{aligned}
$$

Apart from the approximation just mentioned, there are several implicit assumptions. We have ignored the possibility that the Huang-Rhys factor $S$ may differ for emission and absorption because the effective frequencies are so similar in the ground and excited states. In addition, it is possible that emission may take place from a different excited state from the one involved in absorption. Such an effect might show up in measurements of the decay time of F-centre emission, and may have been seen in $\mathrm{KCl}$ (Swank and Brown 1963).

The emission band of the $F$ centre has not been identified in magnesium oxide, although Clarke (1957) reported luminescence in the right region of the spectrum. Unfortunately, the effects of other defects, notably transition group ions, will almost certainly complicate the spectrum. In addition the luminescence may be quenched by other defects. Work on pure crystals should be fruitful, although it is perhaps salutary to recall that no emission has been observed in lithium fluoride (Spinolo 1965), the alkali halide most closely resembling magnesium oxide.

\section{Conclusion}

The temperature dependence of the width and position of the $F$ band has been measured in magnesium oxide. The measurements have been interpreted in terms of a simple configuration coordinate diagram. From this we deduce the Huang-Rhys factor $S$, the effective frequencies in the ground and excited states and the probable emission energy. 'The excited-state frequency is smaller then the ground-state frequency, although this is possibly a consequence of the very approximate way the effects of thermal expansion were subtracted in analysing the line shift. In analysing the observed absorption band to separate the effects of impurities three additional bands were found. So far as we are aware they are intrinsic. They appear to be in the right position and of the right strength to be the analogues of the $\mathrm{K}$ and $\mathrm{Y}$, bands in alkali halides. The origin of the lines has not been positively identified.

The results can be summarized by observing that the $F$ centre in magnesium oxide behaves in a very similar way to that in alkali halides. The Huang-Rhys factor is of the same order, and the effective frequency comes in the same region of the spectrum. Even 
the empirical rule connecting the compressibility and effective frequency holds with the same coefficient. The predicted emission energy is near the blue end of the visible spectrum at about $2 \cdot 4 \mathrm{ev}$.

\section{References}

Barron, T. H. K., Berg, W. T., and Morrison, J. A., 1959, Proc. R. Soc. A, 250, 70-83.

Biggers, R. E., Bell, J. T., Long, E. C., and Russ, O. W., 1966, Oak Ridge National Laboratory Rep., No. ORNL 3834.

Clarke, F. J. P., 1957, Phil. Mag., 2, 607-27.

Dexter, D. L., KLICK, C. C., and Russell, G. A., 1955, Phys. Rev., 100, 603-5.

Durrand, M. A., 1936, Physics, 7, 297-8.

Henderson, B., 1966, Br. J. Appl. Phys., 17, 851-4.

Henderson, B., and King, R. D., 1966, Phil. Mag., 13, 1149-56.

Huang, K., and Rhys, A., 1950, Proc. R. Soc. A, 204, 406-23.

Hughes, A. E., Pooley, D., Rahman, H. U., and Runciman, W. A., 1967, A.E.R.E. Rep., No. R5604. JacoBs, I. S., 1954, Phys. Rev., 93, 993-1004.

Johnson, P. D., 1954, Phys. Rev., 94, 845-6.

Kemp, J. C., Ziniker, W. M., and Glaze, J. A., 1966, Phys. Lett., 22, 37.

LÜTY, F., 1960, $Z$. Phys., 60, 1-10.

Markham, J. J., 1959, Rev. Mod. Phys, 31, 956-89.

1966, F-Centres in Alkali Halides (New York: Academic Press).

Peckham, G., 1967, Proc. Phys. Soc., 90, 657-70.

Podini, P., and SpInolo, G., 1966, Proprieta Ioniche ed Elettroniche degli Alogenuri Alcalini (Milan: Milan University Press), pp. 86-91.

Pryce, M. H. L., 1966, Phonons in perfect lattices and in lattices with point imperfections, Ed. R. W. H. Stevenson (Edinburgh: Oliver and Boyd).

Rabin, H., and ReICH, H., 1964, Phys. Rev., 135, A101-10.

Soshea, R. W., Dekker, A. J., and Sturtz, J. P., 1958, J. Phys., Chem. Solids, 5, 23.

Spinolo, G. G., 1965, Phys. Rev., 137, A1495-6.

Spinolo, G. G., and Smith, D. Y., 1965, Phys. Rev., 140, A2117-20, A2121-9.

Stoneham, A. M., 1966, Proc. Phys. Soc, 89, 909-21.

Swank, R. K., and Brown, F. C., 1963, Phys. Rev., 130, 34.

UNRuh, W., and Culvahouse, J., 1967, Phys. Rev., 154, 861-6.

Wertz, J. E., Auzins, P., Silsbee, R., and Weeks, R. A., 1957, Phys. Rev., 107, 1535-7.

Wertz, J. E., Orton, J. W., and Auzins, P., 1961, Discuss. Faraday Soc., 31, 140-50.

Wertz, J. E., Saville, G. S., Hall, L., and Auzins, P., 1964, Proc. Br. Ceram. Soc, 1, 59. 